\title{
Recomendaciones para la creación, cuidado y manejo de los accesos vasculares para hemodiálisis. Documento de Posición del Comité de Nefrología Intervencionista y del Grupo de Consenso para Optimización de Accesos Vasculares de la Sociedad Latinoamericana de Nefrología e Hipertensión
}

\author{
Mauricio Arvizu-Hernández1 , Octavio J. Salgado², Jorge Arellano-Sotelo³, Gerardo Barquero 4 , \\ Yarima Batista ${ }^{5}$, Gerardo G. Corpus ${ }^{6}$, Manuel Llaro $^{7}$, Katty Rabanal $^{8}$, Precil D. Miranda de Menezes ${ }^{9}$, \\ Juan C. García ${ }^{10}$, César Restrepo ${ }^{11}$, Jaime Vélez ${ }^{12}$, Guillermo Rosa-Díez ${ }^{13}$, Neiro Quintero ${ }^{14} y$ \\ Ricardo Correa-Rotter ${ }^{1 *}$
}

${ }^{1}$ Departamento de Nefrología y Metabolismo Mineral, Instituto Nacional de Ciencias Médicas y Nutrición Salvador Zubirán, Ciudad de México, México; ${ }^{2}$ Nefrología Intervencionista, Universidad Católica de Cuenca, Cuenca, Ecuador; ${ }^{3}$ Radiología Intervencionista, Hospital Centro Médico Nacional Siglo XXI, Instituto Mexicano del Seguro Social, Ciudad de México, México; ${ }^{4}$ Servicio de Nefrología, Hospital San Juan de Dios, Caja Costarricense de Seguro Social, San José, Costa Rica; ${ }^{5}$ Servicio de Nefrología, Complejo Hospitalario Metropolitano Caja de Seguro Social, Ciudad de Panamá, Panamá; ${ }^{6}$ Servicio de Nefrología y Hemodiálisis, Centro de Atención en Nefrología Integral e Intervencionismo Renacer, Saltillo Coahuila, México; ${ }^{7}$ Servicio de Nefrología, Hospital Alberto Sabogal Sologuren, Callao, Perú; ${ }^{8}$ Servicio de Nefrología, Hospital Nacional Guillermo Almenara Irigoyen, Seguro Social de Salud (ESSALUD), Lima, Perú; ${ }^{9}$ División de Nefrología, Hospital das Clínicas da Faculdade de Medicina da USP, São Paulo, Brasil; ${ }^{10}$ Servicio de Nefrología y Urología, Hospital Regional de Alta Especialidad del Bajío, León, Guanajuato, México; ${ }^{11}$ Departamento Clínico, SES Hospital de Caldas, Manizales, Colombia; ${ }^{12}$ Departamento de Cirugía Vascular, Christus Sinergia-Clínica Farallones, Cali, Colombia; ${ }^{13}$ Servicio de Nefrología, Hospital Italiano de Buenos Aires, Buenos Aires, Argentina; ${ }^{14}$ Departamento de Nefrología y Metabolismo Mineral, Hospital Barros Luco, Santiago de Chile, Chile

\section{Resumen}

Este documento establece la posición del Comité de Nefrología Intervencionista y del Grupo de Consenso para Optimización de Accesos Vasculares de la Sociedad Latinoamericana de Nefrología e Hipertensión (SALNH) y es el resultado del interés promovido en la sociedad para diseñar un conjunto de recomendaciones, simples, exhaustivas y sobre todo aplicables a la región acerca de la creación, cuidado y manejo de los accesos vasculares para pacientes en hemodiálisis; para ello se seleccionó la información con mayor sustento científico y que mejor se adapta a las necesidades de los pacientes. Este documento intenta poner al alcance de todos los profesionales de la salud y los pacientes las mejores opciones sobre el acceso vascular (AV) para hemodiálisis, desde su planeación, creación y vigilancia hasta la resolución de las complicaciones potenciales.

Palabras clave: Acceso vascular. Hemodiálisis. Catéter. Fístula arteriovenosa. Recomendaciones. SLANH.

\section{Correspondencia:}

*Ricardo Correa-Rotter E-mail: correarotter@gmail.com
Disponible en internet: 18-06-2021 Nefro Latinoam. 2021;18:58-70 www.nefrologialatinoamericana.com 2444-9032/@ 2021 Sociedad Latinoamericana de Nefrología e Hipertensión. Publicado por Permanyer. Este es un artículo open access bajo la licencia CC BY-NC-ND (http://creativecommons.org/licenses/by-nc-nd/4.0/). 
Recommendations for the creation, care, and management of vascular accesses for hemodialysis. Position Document of the Interventional Nephrology Committee and the consensus group for optimization of vascular accesses of the Latin American Society of Nephrology and Hypertension

\begin{abstract}
This document constitutes a position of the Interventional Nephrology Committee and the Consensus Group for Optimization of Vascular Accesses of the Latin American Society of Nephrology and Hypertension (SALNH) and is the result of the interest promoted in our society to generate a set of practical and applicable recommendations for the region, regarding the creation, care, and management of vascular accesses for patients on hemodialysis. We selected the information with the strongest scientific support and that better adapts to the needs of our patients. This document seeks to make available to all health professionals and patients, the best options regarding Vascular Access (VA) for hemodialysis, from its planning, creation, surveillance, and management of potential complications.
\end{abstract}

Key words: Vascular access. Hemodialysis. Catheter. Arteriovenous fistula. Recommendations. SLANH.

\section{Introducción}

La enfermedad renal crónica (ERC) es hoy en día un problema de salud pública global y uno de los padecimientos de mayores efectos en la población adulta en América Latina. De acuerdo con el último informe del Registro Latinoamericano de Diálisis y Trasplante, presentado en el año 2019 y auspiciado por la Sociedad Latinoamericana de Nefrología e Hipertensión (SLANH), la ERC se ha incrementado en forma progresiva y alarmante en los últimos años; la prevalencia de pacientes con tratamiento de reemplazo renal ha pasado de una tasa de 119 pacientes por millón de habitantes (PMH) en 1991 a 278 pacientes por PMH en el año 2000 y a 660 pacientes por $\mathrm{PMH}$ en el 2013, es decir, un aumento mayor de $550 \%$ en tan sólo 13 años. Se calcula que existen en la región más de 400,000 pacientes con enfermedad renal crónica terminal (ERCT) sometidos a algún tipo de tratamiento de reemplazo renal, hemodiálisis (HD), diálisis peritoneal o trasplante renal. De estas tres modalidades, la más común es la HD y ésta representa más del 50\% (>272,000 pacientes), seguida por el trasplante renal y al final la diálisis peritoneal.

En Latinoamérica, en las últimas dos décadas, se ha registrado un incremento muy notorio del número de pacientes bajo HD; en el año 2006 se consideraba que en la región latinoamericana había alrededor de 300 pacientes con hemodiálisis $\mathrm{PMH}$, con un aumento cercano al 100\% en 12 años hasta alcanzar 544 PMP en el año 2018. Asimismo, es importante reconocer la existencia de una gran heterogeneidad regional, con países con mucho mayor número de pacientes que otros y poblaciones muy pequeñas de pacientes en tratamiento sustitutivo de la función renal; estas disparidades no se explican por diferencias en prevalencia de enfermedad, sino más bien por disponibilidad y acceso'.

El acceso vascular (AV) es un elemento de la mayor importancia para el control adecuado del paciente con HD; su planeación, creación o colocación, uso y vigilancia son aspectos centrales de la atención regular de esta población de pacientes. Por otra parte, el AV es a menudo fuente frecuente de complicaciones, desde procesos infecciosos hasta episodios de disfunción, por lo que el conocimiento de todos los aspectos básicos relacionados con él debe ser una tarea del personal médico y paramédico relacionado.

La valoración previa para la construcción o instalación de un AV, la construcción misma, el cuidado posoperatorio, el manejo común, la punción y el cuidado a largo plazo, entre otras actividades, son tareas multidisciplinarias en las que deben participar de manera activa nefrólogos, cirujanos vasculares, radiólogos intervencionistas, enfermeras, pacientes y familiares.

La mejora de las prácticas de cuidado del AV, con base en las mejores evidencias científicas, adaptadas a las situaciones y aspectos de la región, debe modificar la evolución y desenlaces de los pacientes con ERC en tratamiento de reemplazo renal con HD. Además de la clara necesidad de establecer recomendaciones de práctica clínica para la manipulación del AV, es importante crear grupos de trabajo multicéntricos, nacionales y regionales, para la obtención de evidencias clínicas sólidas sobre mejores prácticas, así como la generación de herramientas de enseñanza y actualización para todo el personal, pacientes y familiares relacionados. 
Latinoamérica no dispone de recomendaciones sobre el control práctico del AV, adaptadas a la región geográfica y la condición sociocultural. Es necesario generar evidencia y recomendaciones que consideren aspectos sociales, económicos, de organización de los sistemas de salud y recursos, que hagan posible la mejor atención disponible para los pacientes.

Se reconoce que existen diferencias en el tipo de AV más utilizado en diferentes regiones del mundo. La fístula arteriovenosa nativa (FAV) es el AV predominante en Europa y gran parte de Asia, a diferencia de lo observado en diversas regiones de América ${ }^{2}$. De las múltiples opciones de $\mathrm{AV}$, se ha demostrado de forma consistente un mejor pronóstico a largo plazo, menor frecuencia de complicaciones y menor mortalidad en pacientes cuyo AV es una FAV, seguida de los injertos arteriovenosos (IAV) heterólogos vasculares y, en tercer lugar, los catéteres, que sin duda alguna son AV importantes en casos de requerimiento inmediato de $\mathrm{HD}$, así como en pacientes con limitaciones para construcción de una FAV o un injerto ${ }^{3,4}$.

\section{Desarrollo y generación de recomendaciones (material y métodos)}

Durante una serie de reuniones de un grupo multidisciplinario latinoamericano de expertos (nefrólogos, cirujanos vasculares, radiólogos intervencionistas), auspiciadas académicamente por el Comité de Intervencionismo de la SLANH, se llevó a cabo una revisión cuidadosa de las mejores guías y prácticas publicadas, y se consensó una serie de recomendaciones y consideraciones relevantes para el control del AV para HD en la región, con la finalidad de crear un documento de recomendaciones de uso práctico. Además de la experiencia personal de los expertos convocados, estas recomendaciones se contrastaron con lo ofrecido por diversas guías de atención del AV para la HD, en particular las guías españolas ${ }^{5}$ y la actualización KDIGO del año $2019^{6}$. Este documento no pretende ser un listado exhaustivo de todos los posibles escenarios y situaciones relacionados con el AV; sin embargo, sí ofrece recomendaciones prácticas y aplicables en la región, con la intención de mejorar las prácticas y el estado actual del manejo del AV de la HD.

Se enlistan recomendaciones en forma numérica progresiva y se abordan aspectos diversos, entre ellos la construcción, el uso y los cuidados del AV del paciente en HD. Se reconoce que las recomendaciones ofrecidas no agotan en forma exhaustiva todos los escenarios y problemas potenciales en esta población de enfermos, pero sí proporciona un análisis de los aspectos más frecuentes y relevantes.

\section{Resultados}

\section{Recomendación 1}

Cuando sea necesario crear o instalar un AV en pacientes que necesitan o requerirán en el mediano y corto plazos tratamientos de reemplazo renal con $H D$, se recomienda considerar siempre que sea posible la creación de una FAV nativa o autóloga.

Es indispensable tratar de ofrecer siempre el mejor AV posible a los pacientes. La evidencia actual apoya que el mejor AV es la FAV; su construcción es relativamente sencilla y su uso reduce la frecuencia de complicaciones infecciosas y no infecciosas a mediano y largo plazo, además de proporcionar un AV cuyo uso puede prolongarse por periodos de tiempo más largos, en comparación con otros tipos de AV. El mayor beneficio se observa cuando se construye la FAV antes de la diálisis y el paciente puede iniciar el tratamiento de reemplazo renal una vez que esta FAV madura ${ }^{7}$. Para contar con una FAV madura al inicio de la HD es pertinente la referencia temprana del paciente al nefrólogo y al cirujano vascular experto en su construcción. En todos los casos se considera ideal que el paciente se refiera al menos ocho a 12 semanas antes de que sea necesario el inicio de la HD crónica.

La calidad de vida, funcionalidad y otros aspectos de los pacientes son mejores cuando se dispone de una FAV o un IAV, en comparación con un catéter ${ }^{8,9}$, y pueden atenuar la morbimortalidad y mejorar la supervivencia de los pacientes en HD (infecciones, hospitalizaciones, episodios cardiovasculares y muerte) ${ }^{10}$.

Por último, en términos de la eficiencia para el tratamiento de la HD, la FAV posibilita mejores flujos sanguíneos y mejor dosis dialítica, lo que modifica en grado positivo los desenlaces a corto, mediano y largo plazos ${ }^{11}$.

\section{Recomendación 2}

Se recomienda que más del $80 \%$ de los pacientes prevalentes y con más de tres meses desde el inicio del tratamiento de reemplazo renal con HD cuenten con un AV definitivo del tipo FAV nativa funcional o en maduración o, en su defecto, un IAV.

Es importante considerar que, aun cuando algunos pacientes tienen que iniciar el tratamiento de reemplazo renal con un AV transitorio del tipo del catéter por las condiciones de presentación de la enfermedad 
renal condicionante o una referencia tardía, debe considerarse el cambio de este AV a una FAV o un IAV a la brevedad posible.

Es tarea del equipo nefrológico encargado dirigir los esfuerzos necesarios para este fin, sin perder de vista los beneficios de los AV definitivos (FAV e IAV) y el mayor riesgo de morbimortalidad relacionado con la permanencia con un catéter. El grupo con mejor pronóstico en sobrevida lo constituyen los pacientes que inician y se mantienen con una FAV funcional y en segundo lugar de mortalidad se ubican los enfermos que inician con una FAV y con posterioridad, por falla de ésta, cambian a un catéter ${ }^{12}$.

Es una tarea del equipo de profesionales de la unidad de HD (nefrología y enfermería) alentar la creación temprana de una FAV en los pacientes con ERC avanzada (KDIGO G5), siempre y cuando la probabilidad de éxito justifique el procedimiento y los riesgos de la construcción, y se descarten las contraindicaciones habituales. Se han desarrollado diferentes iniciativas nacionales y regionales con esta finalidad; el ejemplo más claro es la Fistula First, que ha promovido el grupo KDOQI en Estados Unidos, la cual buscaba alcanzar de manera inicial un $50 \%$ de FAV en los AV incidentes y $40 \%$ en los prevalentes ${ }^{13}$; después se intentó alcanzar una tasa de $64 \%$ de FAV en todos los pacientes en HD. Todos estos esfuerzos aún no han conseguido los objetivos esperados, pero sí han incrementado de forma notoria el número de pacientes que tienen una FAV a lo largo del tiempo. En el caso de Latinoamérica, la proporción de pacientes con FAV es muy variable, pero en general es claro que hay en la mayoría de los países de la región una enorme oportunidad de mejorar, por lo que es necesario un esfuerzo considerable para incrementar el número de individuos en HD con FAV funcional.

Un grupo de pacientes en los cuales no sólo no deben olvidarse estos esfuerzos sino redoblarse, es el de los enfermos que ya se encuentran bajo HD y la reciben a través de un catéter y el grupo de pacientes que poseen una FAV disfuncional y en quienes se espera la pérdida del AV en los siguientes meses. En este grupo de individuos es necesario establecer un plan para favorecer la creación de un segundo AV permanente, con la finalidad de evitar la necesidad de colocar un catéter y favorecer la maduración de la segunda FAV antes de la pérdida del AV inicial.

\section{Recomendación 3}

Se recomienda promover la construcción de un acceso vascular (AV) permanente en el momento adecuado, antes del inicio de la HD, con la finalidad de que esté maduro para su uso cuando se requiera, preferentemente desde la primera sesión. Esto exige referencia temprana al nefrólogo y cirujano vascular.

Se recomienda considerar a los pacientes con ERCT para la construcción de una FAV, cuando la tasa de filtración glomerular calculada (TFGe) sea $<15 \mathrm{ml} / \mathrm{min} / 1.73 \mathrm{~m}^{2} \mathrm{SC}$.

En pacientes con ERC y rápida progresión se recomienda considerar la preparación a partir de $<25 \mathrm{ml} /$ $\mathrm{min} / 1.73 \mathrm{~m}^{2} \mathrm{SC}$. En pacientes con ERC y deterioro lento o nulo, la referencia para la creación de la FAV puede retrasarse a tasas $<10 \mathrm{ml} / \mathrm{min} / 1.73 \mathrm{~m}^{2} \mathrm{SC}$.

Hasta el día de hoy no se cuenta con una herramienta infalible para predecir cuándo será necesario el inicio de un tratamiento de reemplazo renal si el paciente se encuentra ya en ERC de estadios G4 a G5. Algunos informes de series de casos han considerado la creación de una FAV con base en la probabilidad de inicio del tratamiento de reemplazo de la función renal según sea la TFGe, con resultados diversos; en algunos de ellos, sólo el $57 \%$ de los pacientes en quienes se crea una FAV la utilizó para HD en los siguientes 12 meses después del procedimiento ${ }^{14}$. Sin embargo, se considera que un factor importante es la pendiente calculada de deterioro de la TFGe; los pacientes con deterioros acelerados, valores más elevados del índice proteinuria/creatinuria y hospitalizaciones frecuentes con episodios de lesión renal aguda $(\mathrm{LRA})^{15}$ se pueden beneficiar de la creación de una FAV aun con TFGe mayores.

\section{Recomendación 4}

Deben considerarse la edad, la funcionalidad y la opinión del paciente para la construcción de un AV, sobre todo en pacientes de edad avanzada, con múltiples comorbilidades y con expectativa de vida limitada a corto o mediano plazo.

Esto es aplicable a todos los pacientes; sin embargo, es obligación del equipo de profesionales responsables notificar apropiadamente los beneficios claros de un AV permanente, en particular de la construcción de una FAV.

De manera adicional, como parte integral de la valoración anterior a la creación de una FAV, deben considerarse todos los aspectos relacionados con el paciente mismo y que puedan modificar el desenlace del AV. Es importante reconocer las comorbilidades presentes; en especial relevante es el conocimiento de la afección cardiovascular y otras anomalías que modifiquen de forma sustancial la expectativa de vida del paciente, como la posibilidad de alguna enfermedad neoplásica. 
En el caso de insuficiencia cardiaca confirmada o sospecha, debe completarse el estudio del paciente con una valoración cardiológica. En algunos pacientes, una FAV puede ser inapropiada al inducir "secuestro del gasto cardíaco y mayor sobrecarga por aumento del retorno venoso" en pacientes con fracción de expulsión limitada.

Además de las anomalías médicas, deben reconocerse y considerarse las alteraciones de la funcionalidad, aspectos sociales e incluso el pronóstico de vida del paciente; tal y como se recomienda en las últimas guías de KDOQI del acceso vascular ${ }^{6}$, debe realizarse un "plan de vida" que incluya estos aspectos y crear o colocar el AV ideal para ese paciente, dadas sus condiciones generales e individuales. Esto implica sin excepción que, en algunos pacientes con esperanza de vida intrínsecamente limitada, puede justificarse el uso de un acceso de tipo catéter tunelizado de uso prolongado, con la finalidad de evitar los procesos vinculados con la maduración del acceso, reducir al mínimo molestias y evitar potenciales complicaciones.

\section{Recomendación 5}

Se recomienda que, en condiciones ideales, se refiera a los pacientes y se los valore en fase temprana (al menos ocho a 12 semanas antes del requerimiento de la $H D$ ) con el equipo médico-quirúrgico encargado de la creación de la FAV.

El grupo considera importante reiterar la referencia temprana al equipo de cirugía vascular como parte de la valoración para la creación del AV; no existe en las publicaciones médicas evidencia acerca del tiempo mínimo para dicha referencia ${ }^{16}$, pero sí datos indirectos de que la referencia anticipada se relaciona con una mayor probabilidad de iniciar HD con una FAV madura; dicha probabilidad se vincula con el tiempo de referencia al nefrólogo ${ }^{17,18}$ y el equipo quirúrgico. Los pacientes con ERC en etapas G5, y en algunos casos algunos con estadio G4 de rápida progresión, deben referirse al equipo multidisciplinario (nefrología y cirugía vascular) lo antes posible para la creación de una FAV de tal manera que pueda construirse idealmente unos cuatro a seis meses antes de la necesidad de iniciar HD, lo que hará posible su adecuada maduración y viabilidad funcional al momento de requerirse el inició de este tratamiento sustitutivo.

Una parte esencial de la preparación del paciente para la creación del AV es la asesoría que reciba el paciente y sus familiares respecto de los cambios estéticos, fisiológicos y posibles repercusiones del procedimiento (tasas de éxito o falla), entre otros factores, de tal modo que el paciente pueda contar con la mayor información posible, lo cual modificará de manera positiva los desenlaces del procedimiento.

\section{Recomendación 6}

Es indispensable el conocimiento anatómico del sistema arterial y venoso y de las áreas apropiadas para la creación de la FAV por parte de todo el equipo médico participante.

El conocimiento de la anatomía, así como de las características necesarias para la creación de una FAV en pacientes con ERC desde estadios moderadamente avanzados, favorece el cuidado de las estructuras vasculares y mejora el pronóstico del AV.

Se recomienda llevar a cabo una valoración inicial mediante la exploración física enfocada en los pulsos arteriales, los datos de la perfusión periférica, el sistema venoso visible y palpable, la dilatación con la aplicación de un torniquete y la ausencia de edema. Estos conocimientos debe dominarlos y compartirlos el personal médico (nefrología y cirugía vascular) y el de enfermería para que todos los integrantes del equipo sean capaces de identificar las venas apropiadas y las características necesarias para la creación de una FAV. La falta de conocimiento de las características anatómicas y funcionales relevantes de la vasculatura de las extremidades puede producir a mediano plazo acciones que propicien la falla posterior de la FAV o impidan que ésta pueda utilizarse como AV para la HD. Un ejemplo relevante es la preservación de la circulación venosa idónea de brazos (véase la recomendación 7), con la menor cantidad posible de punciones venosas en el tiempo anterior a la necesidad de construir una FAV.

En el caso de los catéteres, cuando éstos sean la vía requerida para iniciar $\mathrm{HD}$, es importante no colocar este tipo de accesos (tunelizados o no) en venas con alto riesgo de trombosis o estenosis, como las venas subclavias, innominada izquierda, y otras más. La colocación de catéteres en estos sitios puede influir de manera adversa en la creación posterior de una FAV en extremidades superiores. En los pacientes en quienes no es posible evitar tal procedimiento se recomienda su identificación y cambio a la brevedad posible a una vena de menor riesgo.

\section{Recomendación 7}

En los pacientes con ERC de estadios G3 a G5 siempre se recomienda la preservación de venas en sitios susceptibles para la creación de FAV. 
En relación con esta recomendación, se emiten las siguientes puntualizaciones:

1. Las venas dorsales de la mano son el lugar preferido para las flebotomías y los accesos venosos periféricos para infundir de forma transitoria soluciones 0 fármacos.

2. Las venas yugulares internas son el lugar preferido para la instalación de un acceso venoso central de tipo catéter, tunelizado o no.

3. Las venas yugulares externas son una alternativa aceptable para el acceso venoso central de tipo catéter, tunelizado o no.

4. Las venas subclavias no deben usarse para el acceso venoso central de tipo catéter, a menos que sea el único abordaje viable. Una vez más: no deben usarse para un AV inicial antes de agotar otras opciones.

5. Debe evitarse la colocación de catéteres centrales de inserción periférica (PICC).

6. Las principales venas que deben protegerse son:

(a) Vena cefálica en todo su trayecto, desde la articulación de la muñeca hasta la región deltoidea.

(b) Vena basílica en toda su extensión, desde la muñeca hasta la región de la axila.

Estos vasos (venas cefálica y basílica) son los apropiados de la extremidad superior para la creación de una FAV. Otras venas del antebrazo, como las venas dorsales de la mano, la mediana antecubital y las venas colaterales del antebrazo, no se utilizan para la construcción de FAV y, en última instancia, pueden usarse para punciones venosas; se recomienda que las punciones se realicen en el brazo dominante, con agujas del menor calibre posible, por el personal de mayor experiencia del equipo, y que se reduzca al mínimo el tiempo de estancia del catéter.

Las venas deben protegerse de manera bilateral y no sólo las del miembro superior no dominante. Las punciones repetidas del sistema venoso periférico crean áreas de estenosis o esclerosis que alteran de modo negativo el éxito de la creación y maduración de las FAV. Más aún, se considera riesgosa la colocación de catéteres centrales de inserción periférica ${ }^{19}$, catéteres venosos centrales, marcapasos, introductores para dispositivos de vigilancia cardiaca, etc. Por último, deben evitarse las punciones arteriales (p. ej., para gasometría) del antebrazo.

Pueden promoverse algunas iniciativas que han demostrado utilidad, entre ellas la de Save your vein (Salva tu vena), impulsada por los Institutos de Salud del Reino Unido, enfocadas en el cuidado y la preservación del sistema venoso periférico en pacientes con
ERC. Estas iniciativas buscan crear conciencia de la importancia del sistema venoso periférico y se acompañan de la entrega de una tarjeta de identificación con recomendaciones de toma de muestras sanguíneas de las venas del dorso de la mano, alternar sitios de punción o evitar las punciones innecesarias ${ }^{20}$.

\section{Recomendación 8}

Se recomienda siempre considerar como primer sitio de construcción de una FAV el miembro superior no dominante $y$ en un punto lo más distal posible.

Se recomienda la siguiente secuencia de accesos vasculares definitivos (FAV):

1. FAV radiocefálica

2. FAV cubitobasílica

3.FAV humerocefálica

4. FAV humerobasílica (con superficialización)

5. FAV húmero-humeral

6. Prótesis húmero-axilar

7. Variantes en miembro inferior

La valoración y las decisiones para la creación de una FAV exitosa implican una visión multidisciplinaria y holística (nefrología, enfermería, cirugía vascular, pacientes, otros) para poder tomar la mejor decisión posible. De nueva cuenta: el mejor AV es la FAV y siempre que sea posible y apropiado debe considerarse como primera opción.

Una manera de asegurar la disponibilidad en el tiempo de sitios para crear uno o más AV consiste en seguir un orden de creación que considere aspectos como el lado no dominante (en la medida de lo posible) y el inicio lo más distal posible en la extremidad superior, con la intención de aprovechar el mayor número y localización de sitios posibles para futuras FAV. La FAV radiocefálica es la mejor opción inicial y debe considerarse bilateral. En algunos pacientes, la valoración inicial por parte del experto, clínica y ultrasonográfica, hace poco viable el éxito con la radiocefálica y por tanto debe considerarse desde ese momento la construcción de una FAV cubitobasílica o humerocefálica.

\section{Recomendación 9}

La adecuada valoración preoperatoria integral del paciente con ERC, antes de la creación de una FAV, incrementa la probabilidad de éxito, disminuye el riesgo de complicaciones y garantiza la seguridad del paciente durante y después del procedimiento.

Deben identificarse los aspectos clínicos mínimos indispensables para que un paciente pueda someterse 
con seguridad al proceso de creación de una FAV. Algunos aspectos relevantes son: el paciente no debe mostrar datos de insuficiencia cardíaca ni signos de infección activa de cualquier tipo, idealmente en peso seco, con presión arterial mínima de pie de 110/70 mmHg, frecuencia cardíaca de 60 a 95/min, con suspensión oportuna del tratamiento antitrombótico 0 anticoagulación según la consideración del equipo quirúrgico, con hemoglobina $>7.5 \mathrm{~g}$ para procedimientos ambulatorios, sin leucocitosis ni alteraciones de número de las plaquetas, en condiciones ideales con tiempos de coagulación normales y en caso de recibir tratamiento con corticoesteroides con dosis menores de $20 \mathrm{mg} /$ día de prednisona o su equivalente. Es importante también conocer si existen factores inmunológicos protrombóticos, como los anticuerpos antifosfolípidos $u$ otros.

Cuando el paciente se encuentra ya en HD con un catéter y se envía a construcción de una FAV, debe recibir una sesión de HD 24 horas antes del procedimiento quirúrgico.

\section{Recomendación 10}

Se recomienda mantener el apropiado seguimiento posoperatorio de potenciales complicaciones luego de la construcción de la FAV con exploración física y, en condiciones ideales, cuando sea posible, con ultrasonido Doppler.

La vigilancia temprana después de la creación de FAV es fundamental para identificar datos de alarma y favorecer un mejor seguimiento de la maduración del acceso.

Los objetivos más importantes deben ser la permeabilidad de la FAV, la evolución del diámetro de la vena y la identificación de áreas de hiperplasia de la íntima o zonas de estenosis. El reconocimiento de cualquier alteración en uno de estos aspectos debe obligar a tomar medidas correctivas o vigilar de manera más estrecha, de acuerdo con el caso.

La vigilancia puede dividirse en dos etapas, según sean las complicaciones posibles:

- Fase temprana: trombosis, sangrado, infección, seromas, linfoceles, etc.

- Fase tardía: trombosis, aneurismas, falla en la maduración, síndrome de secuestro, síndrome de hipertensión venosa, etc.

Al final, con base en los hallazgos identificados, se puede recomendar el ajuste de los fármacos y en algunos casos el uso de antibióticos, antitrombóticos o alguna intervención quirúrgica correctiva según corresponda.

\section{Recomendación 11}

Se recomienda establecer procedimientos de seguimiento y promoción de la maduración de la FAV.

Para FAV radiocefálicas o cubitobasílicas el ejercicio de compresión con la mano de una esfera de goma o pelota puede acelerar la maduración de la FAV (aumento del calibre venoso y el flujo); el momento de inicio es variable, pero por lo general se recomienda después de transcurrida la primer semana desde la creación de la fístula; en el caso de fístulas humerocefálicas o humerobasílicas, el ejercicio inicial puede ser también la compresión de una esfera de goma y debe incluirse la flexión del bíceps con una pesa hasta de $1 \mathrm{~kg}$; y se debe educar al paciente en relación con la importancia de las repeticiones sobre la fuerza del ejercicio.

En el periodo posoperatorio debe realizarse lo siguiente:

- Vigilar la presión arterial, evitar los episodios de hipotensión durante y después de la diálisis y buscar como objetivo presiones $\sim 140 / 90 \mathrm{mmHg}$ las primeras tres a cuatro semanas, en algunos casos.

- Evaluar el ajuste de los fármacos antihipertensivos para evitar episodios de hipotensión.

- Evaluar el ajuste del peso seco de manera transitoria para prevenir episodios de hipotensión; es prudente incrementar el peso seco (p. ej., 1 kg) de tal manera que se alcancen dichas cifras, con vigilancia estrecha de los episodios de sobrecarga hídrica.

- Evitar vendajes circulares ajustados.

- Evaluar cambios en la cicatriz quirúrgica (enrojecimiento, secreción, aumento de volumen en anastomosis).

- Vigilar y detectar el desarrollo de edema que no mejora luego de las primeras tres semanas; en estos casos debe investigarse oclusión venosa distal o central o un incremento del flujo a través de la anastomosis de la FAV.

\section{Recomendación 12}

Se recomienda el inicio de uso de una FAV o un IAV cuando se confirme la maduración de la FAV o pase el tiempo indicado por el equipo quirúrgico para la punción del IAV.

El momento apropiado para puncionar una FAV ya construida puede ser variable, pero en promedio es de 
seis a ocho semanas. Debe reiterarse que cada caso debe valorarse de modo individual para ese fin.

La valoración de la maduración de la FAV puede efectuarse en muchos casos mediante exploración física si el personal tiene amplia experiencia, pero en otros casos es necesario un mapeo ultrasonográfico Doppler, que confirme que el flujo y el calibre venoso son apropiados para la función. En este sentido, se ha empleado una "regla de los 6" como un instrumento que permite determinar mejor la madurez de la FAV ( 6 semanas de construida, longitud del trayecto arterializado de al menos $6 \mathrm{~cm}$, flujo sanguíneo de al menos $600 \mathrm{~cm}^{3} / \mathrm{min}$, diámetro $>0.6 \mathrm{~cm}$ y profundidad desde la piel aproximada de $0.6 \mathrm{~cm}$ ). La canulación de un IAV en general es posible con seguridad al cumplirse tres semanas de construido el acceso.

Un aspecto fundamental es que la punción inicial de una FAV se inicie, como se ha mencionado ya, a partir de la semana sexta a octava después de su construcción, y la realice personal entrenado y con amplia experiencia en el procedimiento. Si el paciente tiene un catéter funcional y la FAV sustituirá a ese AV temporal, la utilización de la FAV se debe introducir de forma gradual, al combinar el uso del catéter y la FAV; y se inicia con la línea venosa, lo cual favorece la maduración de la FAV. Se recomienda mantener el catéter hasta colocar ambas agujas (arterial y venosa) en la FAV en tres oportunidades, sin incurrir en incidentes y con el mayor calibre posible (15-16 G) que permita alcanzar los flujos indicados antes de retirar el catéter con seguridad.

Durante el uso de la FAV se recomienda elegir las punciones mediante una técnica de forma escalonada, sobre las otras técnicas (en ojal). Se pueden utilizar ungüentos con base de lidocaína para facilitar las primeras punciones y reducir el malestar generado por ellas, lo que facilita el proceso de inicio de uso de la FAV.

La canulación de la prótesis vascular (injerto) se basa en el tipo de injerto utilizado; si es de paredes normales, las primeras punciones se efectúan a las cuatro semanas, pero si se trata de paredes con triple capa, las punciones pueden iniciarse después de una semana tras su colocación, siempre y cuando no existan complicaciones.

\section{Recomendación 13}

Se recomienda la vigilancia (estudio clínico) y seguimiento (vigilancia instrumentada) de la función del acceso vascular.
Es necesario mantener una vigilancia estrecha del funcionamiento de la FAV, que permita la identificación oportuna y el tratamiento temprano de las posibles complicaciones. El control temprano de dichas complicaciones eleva la probabilidad de éxito de la preservación y en algunos casos la posibilidad de corrección o reparación para preservar un AV funcional. Se recomienda establecer programas de vigilancia de manera periódica basados en la medición y vigilancia de los cambios de parámetros, como presiones dinámicas y estáticas determinadas por la máquina de HD, flujo máximo alcanzado (considerar como considerable un descenso del $25 \%$ ), tiempo de sangrado, parámetros para la medición de la dosis de diálisis y recirculación; en los casos en los que existan cambios clínicos indicativos de disfunción se recomienda complementar el abordaje mediante estudios basados en ultrasonido, medición de flujos, etc. ${ }^{21-23}$.

Es importante valorar de manera periódica los cambios potenciales de las FAV, los más importantes son el desarrollo de vasos colaterales, la formación de aneurismas y seudoaneurismas, la aparición o desarrollo de sitios de estenosis en la porción arteriolizada de la vena o los vasos centrales, así como el desarrollo de fístulas de alto flujo (>1,500 $\mathrm{ml} / \mathrm{min})$, al igual que los potenciales cambios cutáneos relacionados con el uso de la FAV.

\section{Recomendación 14}

Cuando se requiera el inicio inmediato de tratamiento de reemplazo renal con HD y se conoce que el paciente es portador de ERC, se recomienda la colocación de un catéter tunelizado que, en condiciones ideales, debe colocarse en primera instancia en la vena yugular interna derecha (primera opción).

En los pacientes con ERC e indicaciones claras o de urgencia para iniciar tratamiento de sustitución con HD (uremia, hiperpotasemia, acidosis, sobrecarga de volumen, etc.), y en quienes no es factible esperar al proceso de construcción y maduración de una FAV, el AV debe ser un catéter (tunelizado de preferencia) con la finalidad de resolver la urgencia. Se reconoce que el riesgo de infecciones, disfunción y trombosis puede ser mayor con los catéteres no tunelizados o temporales, pero es claro que la colocación de un catéter tunelizado requiere, además del insumo específico, destrezas en el equipo médico para su instalación, equipo de ultrasonido, una sala de fluoroscopia, etc., por lo que en algunos casos se instalará un catéter no tunelizado ${ }^{24,25}$. De manera ideal se recomienda contar 
con personal médico y de enfermería con experiencia en el procedimiento, así como el equipo y material necesario para la instalación de dichos catéteres tunelizados, dado que han demostrado su utilidad en la atención de los pacientes crónicos en HD, lo cual ha permitido alcanzar adecuados flujos sanguíneos y tasas relativamente bajas de disfunción y otras complicaciones ${ }^{26,27}$.

Para la colocación apropiada de un catéter tunelizado es necesario considerar algunos aspectos técnicos que favorezcan su adecuado funcionamiento: realizar la punción de acuerdo con la técnica del abordaje posterior en el caso de las venas yugulares; longitud del catéter (depende del sitio de abordaje y estatura del paciente); localización de la punta en el interior de la aurícula derecha; prevención del acodamiento del catéter en el trayecto del túnel; el sitio de salida del catéter debe localizarse en sentido inferior, en la región del tórax, por debajo de la clavícula; la distancia del sitio de salida al cojinete/retenedor debe ser al menos de $1.5 \mathrm{a} 2 \mathrm{~cm}$. En consecuencia, se reitera que es necesario que el operador tenga el conocimiento y la experiencia requerida ${ }^{28}$.

En los pacientes con ERC en los que se coloca un catéter tunelizado debe elegirse el sitio de inserción en el siguiente orden: yugular interna derecha, yugular externa derecha, yugular interna izquierda, yugular externa izquierda y catéteres femorales.

En pacientes con dificultades para la colocación de un catéter en el sistema venoso superior se ha notificado la colocación de catéteres tunelizados largos mediante un abordaje femoral (punta en aurícula derecha) con resultados positivos ${ }^{29}$.

Si no es posible la inserción de un catéter tunelizado se coloca un catéter temporal no tunelizado, siempre con el objetivo ulterior de no permitir su permanencia por más de dos semanas ${ }^{6}$.

\section{Recomendación 15}

Se recomienda que la colocación de los AV de tipo catéter (tunelizado o no) se realice bajo control ultrasonográfico en tiempo real y por personal con la experiencia necesaria.

La colocación del AV bajo guía ultrasonográfica permite reducir el número y la gravedad de las posibles complicaciones, así como el tiempo del procedimiento mismo. El uso del ultrasonido permite identificar la anatomía y valorar la permeabilidad vascular, facilita la punción dirigida del vaso y puede ser de utilidad en la identificación de algunas complicaciones.
Cuando se considera la canulación de la vena yugular interna, el uso del ultrasonido ha demostrado mejorar la tasa de éxito del procedimiento, en comparación con la técnica basada en referencias anatómicas. Con el ultrasonido se logra la canulación apropiada en los dos primeros intentos en cerca del $80 \%$ de los casos, con una reducción del número de intentos (punciones) y la tasa de complicaciones, como punción arterial, formación de hematoma y otras en casi $50 \%^{30}$.

Se recomienda el uso de equipos de ultrasonido que cuenten con la función de imágenes bidimensionales y Doppler, así como el uso de transductores lineales de alta frecuencia $(5-15 \mathrm{MHz})$ con una superficie de escaneo de 20 a $50 \mathrm{~mm}$; estos transductores hacen posible una alta definición de la imagen a costa de poca profundidad ${ }^{31}$.

Se recomienda que la capacitación en el uso de ultrasonido se generalice en los centros de atención de pacientes con ERC con requerimientos de HD, y en los centros de formación del personal médico (nefrólogos) y paramédico (enfermería).

\section{Recomendación 16}

En los casos en los que no exista la posibilidad anatómica de crear una FAV o colocar un IAV, así como ante agotamiento de los AV, se considera como opción a largo plazo la permanencia del AV tunelizado.

Se sugiere la utilización de un catéter venoso central tunelizado como AV definitivo en algunas circunstancias especiales: enfermedad vascular periférica grave, hipotensión arterial crónica, esperanza de vida $<6$ meses, cardiopatía grave con función ventricular izquierda deprimida y deseo expreso del paciente a pesar de ser informado en forma apropiada de las opciones y ventajas de la FAV o el IAV.

Los AV tunelizados son la opción recomendada para sustituir a una FAV o un IAV, en algunos pacientes con ciertas características ya descritas. El tiempo de permanencia de un AV tunelizado puede ser de varios meses, sin representar un riesgo de otras complicaciones. Los catéteres tunelizados suelen estar fabricados de materiales de mayor biocompatibilidad que los temporales. Debido a su calibre, suministran con frecuencia flujos cercanos a lo observado con el uso de una FAV ${ }^{22}$ $y$, debido al diseño de la punta, algunos reducen al mínimo la tasa de recirculación durante el tratamiento de $\mathrm{HD}^{32,33}$. Un aspecto relevante es su menor tendencia a originar procesos infecciosos y complicaciones trombóticas, en comparación con los catéteres no tunelizados. Algunos estudios han demostrado provocar una 
morbimortalidad intermedia entre la de una FAV (la más baja) o la de los IAV (mejores) y la de los catéteres no tunelizados, estos últimos con la morbimortalidad más alta. Sin embargo, algunos informes han encontrado una tasa elevada de complicaciones en catéteres tunelizados, como disfunción (27\%), estenosis $(33 \%)$, infecciones $(29 \%)$, trombosis $(8 \%)$, y hasta el $50 \%$ requirió un cambio del catéter ${ }^{34}$, por lo que no pueden dejarse de lado estas complicaciones y las acciones necesarias para su prevención.

\section{Recomendación 17}

El empleo de los catéteres temporales no tunelizados debe limitarse a pacientes con insuficiencia renal aguda o con una emergencia de vida que exija su instalación en forma expedita para recibir una HD inmediata.

Se recomienda limitar el tiempo de permanencia de este tipo de accesos a no más de dos semanas; en caso de ser necesario, se determina si debe reponerse por retraso en la recuperación de la función renal y, en el caso de falta de ésta, debe determinarse el AV permanente para cada paciente. Si no es factible la creación de un AV definitivo (FAV o IAV), se considera el cambio por un catéter tunelizado lo más pronto posible.

Se reconoce que el catéter no tunelizado representa el mayor riesgo de complicaciones infecciosas entre todos los AV, así como de disfunción e incluso se ha relacionado con un riesgo mayor de mortalidad, por lo que debe evitarse su permanencia más allá del tiempo recomendado.

\section{Recomendación 18}

Antes de la construcción o instalación de un AV, como una FAV o la colocación de IAV para inicio de $H D$ crónica, debe realizarse una valoración clínica completa por parte del equipo médico encargado del control del AV.

Se debe incluir al menos lo siguiente: historia clínica completa, historial de punciones venosas y catéteres venosos centrales; exploración física minuciosa de ambos brazos, incluida la valoración del sistema venoso y arterial, oximetría de pulso, búsqueda de signos de insuficiencia arterial (prueba de Allen), búsqueda de circulación venosa colateral, y confirmación de sitios potenciales para la creación de una FAV.

En algunos pacientes, la valoración clínica no puede determinar la idoneidad del sistema vascular para la creación de una FAV y deben referirse a la brevedad para estudio complementario que incluya un mapeo con ultrasonido Doppler del sistema venoso y arterial, con valoración de los diámetros, flujos y trayectos vasculares; se buscará que los diámetros vasculares sean, en el caso de la vena, de $3 \mathrm{~mm}$, con una distensibilidad de al menos $30 \%$, y en la arteria de 3-4 mm, sin evidencia de calcificaciones extensas, para considerarlas aptas para la creación de la FAV; debe revisarse la localización y distancia entre la vena y arteria potencialmente utilizables para la creación de la FAV, la distancia entre ambas (factibilidad quirúrgica), así como la variabilidad del diámetro venoso con la aplicación de un torniquete. En algunos casos puede requerirse de flebografía periférica o central o angiotomografía de venas centrales, en particular cuando existe sospecha o cualquier manifestación de oclusión/estenosis venosa.

En pacientes con características limítrofes se recomienda favorecer y recomendar la realización de ejercicios antes de la creación de la FAV, de tal manera que se favorezca la probabilidad de resultados positivos después de la creación de la FAV.

\section{Recomendación 19}

Existe una población con AV convencionales agotados y que pueden considerarse en la categoría de "accesos complejos". La construcción/colocación de un acceso complejo requiere la valoración cuidadosa del comité de AV con amplia experiencia en procedimientos de esta naturaleza (nefrología, cirugía vascular y radiología intervencionista).

Es necesario que un grupo multidisciplinario con experiencia en la atención de casos similares valore al paciente con agotamiento de accesos vasculares para tomar la mejor decisión posible en cada caso.

\section{Recomendación 20}

La educación y el entrenamiento óptimos de todos los equipos y personas que intervienen en la construcción y manipulación de los AV son un instrumento fundamental para el éxito de los programas con objeto de optimizar los resultados clínicos de los pacientes que requieren un $A V$ en $H D$.

\section{Recomendación 20.1}

Se recomienda la creación y realización permanente de cursos de entrenamiento y educación 
continua en nefrología intervencionista para médicos nefrólogos y médicos nefrólogos en formación.

La actualización continua en el área de nefrología intervencionista para personal de nefrología, formado y en formación, hace posible un mejor control del AV, al reducir complicaciones y morbimortalidad y favorecer la creación, mantenimiento y cuidado del AV permanente. Debe hacerse énfasis en el trabajo multidisciplinario requerido y con una visión de largo plazo que incluya la creación de comités del AV en HD.

\section{Recomendación 20.2}

Se recomienda la creación y realización permanente de cursos de entrenamiento y educación continua para el personal de enfermería y técnico en $H D$, en todos los aspectos relativos a la vigilancia, construcción, manipulación y uso de los AV en HD.

El personal de enfermería y técnico juega un papel fundamental, dado que se encarga de la punción y manejo óptimo del AV permanente o temporal en el paciente bajo HD. Es necesaria su participación en los equipos multidisciplinarios y deben tener representación apropiada en los comités de vigilancia del AV en HD. La actualización continua del personal de enfermería permite una mejor atención de estos pacientes, reduce el número de complicaciones y favorece la creación y mantenimiento de FAV funcionales. Este tipo de actividades posibilita también la actualización continua en áreas de mejora del conocimiento.

\section{Recomendación 20.3}

Se recomienda la creación y realización permanente de cursos informativos y de cuidados en los aspectos pertinentes relativos al AV para los pacientes y sus familiares.

La educación del paciente y sus familiares debe formar parte esencial del control del AV; su participación debe ser activa e informada, con la finalidad de reducir el número de complicaciones y favorecer la oportuna creación, los cuidados óptimos y el mantenimiento de un AV vascular, ya sea que se trate de uno temporal tipo catéter o de uno permanente tipo FAV o IAV. Este tipo de conocimiento puede evitar complicaciones infecciosas, auxiliar a la detección temprana de complicaciones de diversos tipos, por ejemplo todas las complicaciones que pueden causar disfuncionalidad y deterioro del AV a corto, mediano o largo plazos.

\section{Conclusiones}

Con este documento se han emitido recomendaciones sencillas y aplicables al día a día en las prácticas de la hemodiálisis en la región. Esta zona tiene algunas desventajas en comparación con otras del mundo, por lo que resulta necesario redoblar esfuerzos y uniformar las mejores prácticas en todos los pacientes; para alcanzar estos objetivos es indispensable la educación de todo el personal médico y paramédico relacionado, y de los pacientes y familiares que padecen ERC en estadios avanzados (KDIGO etapa IV-V). Los avances deben buscarse de manera progresiva y ordenada; hay que modificar aspectos de la práctica actual como el uso extendido de los catéteres temporales o la pobre tasa de creación de fístulas arteriovenosas. Éstos deben considerarse los aspectos más relevantes a modificar, así como generar una visión en conjunto con los pacientes hacia un plan de vida según sus características clínicas y preferencias, que tenga como resultado la elección del mejor acceso vascular para cada paciente. Deben realizarse esfuerzos para alcanzar las mejores sobrevidas de cada uno de los AV de vida larga, ya sea catéteres tunelizados o FAV. La pérdida y disfunción del AV aún representa uno de los mayores problemas del tratamiento crónico de los pacientes en HD.

\section{Agradecimientos}

Agradecemos a todo el personal de Cuidados Renales Medtronic Latinoamérica, en especial a la Dra. Alejandra González De la Vega por la ayuda brindada.

\section{Conflicto de intereses}

Ricardo Correa-Rotter declara como conflicto de intereses la consultoría y la investigación clínica para AstraZeneca, Novonordisk, GSK, Medtronic, y ser conferencista para AstraZeneca, Abbvie, Boheringer, Janssen y Takeda.

Mauricio Arvizu-Hernández declara como conflicto de intereses la consultoría y la investigación para Medtronic, GSK y Novonordisk, y ser conferencista para 
Roche, Baxter y Medtronic, además del apoyo para la educación para Medtronic Latinoamérica.

El resto de los autores no tiene conflicto de intereses que declarar.

\section{Financiamiento}

Este proyecto fue auspiciado y avalado por la Sociedad Latinoamericana de Nefrología e Hipertensión (SLANH). Asimismo, se realizó gracias a un donativo y apoyo no restringido a la comunidad académica de SLANH por parte de Cuidados Renales Medtronic Latinoamérica.

\section{Responsabilidades éticas}

Protección de personas y animales. Los autores declaran que para esta investigación no se han realizado experimentos en seres humanos ni en animales.

Confidencialidad de los datos. Los autores declaran que han seguido los protocolos de su centro de trabajo sobre la publicación de datos de pacientes.

Derecho a la privacidad y consentimiento informado. Los autores declaran que en este artículo no aparecen datos de pacientes.+

\section{Bibliografía}

1. Registro Latinoamericano de Diálisis y Transplante Renal, Informe 2018 (2019). Congreso de la SLANH. Lima, Perú.

2. Pisoni RL, Young EW, Dykstra DM, Greenwood RN, Hecking E, Gillespie B, et al. Vascular access use in Europe and the United States: results from the DOPPS. Kidney Int. 2002 Jan;61(1):305-16. doi: 10.1046/j.1523-1755.2002.00117.x. PMID: 11786113.

3. Dhingra RK, Young EW, Hulbert-Shearon TE, Leavey SF, Port FK. Type of vascular access and mortality in U.S. hemodialysis patients. Kidney Int. 2001 Oct;60(4):1443-51. doi: 10.1046/j.1523-1755.2001.00947.x. PMID: 11576358

4. Pisoni RL, Arrington CJ, Albert JM, Ethier J, Kimata N, Krishnan M, Rayner $\mathrm{HC}$, et al. Facility hemodialysis vascular access use and mortality in countries participating in DOPPS: an instrumental variable analysis. Am J Kidney Dis. 2009 Mar;53(3):475-91. doi: 10.1053/j.ajkd.2008.10.043. Epub 2009 Jan 15. PMID: 19150158

5. Ibeas J, Roca-Tey R, Vallespín J, Moreno T, Moñux G, Martí-Monrós A et al.; por el Grupo Español Multidisciplinar del Acceso Vascular (GE MAV). Spanish Clinical Guidelines on Vascular Access for Haemodialysis. Nefrología. 2017 Nov;37 Suppl 1:1-191. English, Spanish. doi: 10.1016/j. nefro.2017.11.004. Erratum in: Nefrologia. 2019 Jan - Feb;39(1): 1-2. Erratum in: Nefrologia. 2019 Nov - Dec;39(6):680-682. PMID: 29248052.

6. Lok CE, Huber TS, Lee T, Shenoy S, Yevzlin AS, Abreo K, et al.; National Kidney Foundation. KDOQI Clinical Practice Guideline for Vascular Access: 2019 Update. Am J Kidney Dis. 2020 Apr;75(4 Suppl 2):S1-S164. doi: 10.1053/j.ajkd.2019.12.001. Epub 2020 Mar 12. Erratum in: Am J Kidney Dis. 2021 Apr;77(4):551. PMID: 32778223

7. Ozeki T, Shimizu H, Fujita Y, Inaguma D, Maruyama S, Ohyama $Y$, et al. The type of vascular access and the incidence of mortality in japanese dialysis patients. Intern Med. 2017;56(5):481-485. doi: 10.2169/internalmedicine.56.7563. Epub 2017 Mar 1. PMID: 28250291; PMCID: PMC5399196.

8. Afsar B, Elsurer R, Covic A, Kanbay M. Vascular access type, health-related quality of life, and depression in hemodialysis patients: a preliminary report. J Vasc Access. 2012 Apr-Jun;13(2):215-20. doi: 10.5301/ jva.5000032. PMID: 22139743
9. Kim DH, Park JI, Lee JP, Kim YL, Kang SW, Yang CW, et al. The effects of vascular access types on the survival and quality of life and depression in the incident hemodialysis patients. Ren Fail. 2020 Nov;42(1):30-39. doi: 10.1080/0886022X.2019.1702558. PMID: 31847666; PMCID: PMC6968432.

10. Ravani P, Palmer SC, Oliver MJ, Quinn RR, MacRae JM, Tai DJ, et al. Associations between hemodialysis access type and clinical outcomes: a systematic review. J Am Soc Nephrol. 2013 Feb;24(3):465-73. doi: 10.1681/ASN.2012070643. Epub 2013 Feb 21. PMID: 23431075; PMCID: PMC3582202.

11. Cortez AJ, Paulson WD, Schwab SJ. Vascular access as a determinant of adequacy of dialysis. Semin Nephrol. 2005 Mar;25(2):96-101. doi: 10.1016/j.semnephrol.2004.09.016. PMID: 15791561

12. Yeh LM, Chiu SY, Lai PC. The impact of vascular access types on hemodialysis patient long-term survival. Sci Rep. 2019 Jul 24;9(1):10708. doi: 10.1038/s41598-019-47065-z. PMID: 31341241; PMCID:PMC6656721.

13. III. NKF-K/DOQI Clinical Practice Guidelines for Vascular Access: update 2000. Am J Kidney Dis. 2001 Jan;37(1 Suppl 1):S137-81. doi: 10.1016/ s0272-6386(01)70007-8. PMID: 11229969.

14. Bansal N, He C, Murphy DP, Johansen KL, Hsu CY. Timing of preemptive vascular access placement: do we understand the natural history of advanced CKD?: an observational study. BMC Nephrol. 2013 May 28;14:115. doi: 10.1186/1471-2369-14-115. PMID: 23714195; PMCID: PMC3671964.

15. Goel N, Kwon C, Zachariah TP, Broker M, Folkert VW, Bauer C, et al. Vascular access placement in patients with chronic kidney disease Stages 4 and 5 attending an inner city nephrology clinic: a cohort study and survey of providers. BMC Nephrol. 2017 Jan 17;18(1):28. doi: 10.1186/ s12882-016-0431-3. PMID: 28095805; PMCID: PMC5240209.

16. Murad MH, Sidawy AN, Elamin MB, Rizvi AZ, Flynn DN, McCausland FR, McGrath MM, et al. Timing of referral for vascular access placement: a systematic review. J Vasc Surg. 2008 Nov;48(5 Suppl):31S-3S. doi: 10.1016/j.jvs.2008.08.046. PMID: 19000591

17. Astor BC, Eustace JA, Powe NR, Klag MJ, Sadler JH, Fink NE, Coresh J. Timing of nephrologist referral and arteriovenous access use: the CHOICE Study. Am J Kidney Dis. 2001 Sep;38(3):494-501. doi: 10.1053/ ajkd.2001.26833. PMID: 11532680

18. Al-Jaishi AA, Lok CE, Garg AX, Zhang JC, Moist LM. Vascular access creation before hemodialysis initiation and use: a population-based cohort study. Clin J Am Soc Nephrol. 2015 Mar 6;10(3):418-27. doi: 10.2215/ CJN.06220614. Epub 2015 Jan 7. PMID: 25568219; PMCID: PMC4348683.

19. McLennan G. Vein preservation: an algorithmic approach to vascular access placement in patients with compromised renal function. Journal of the Association for Vascular Access, 2007. 12(2), 89-91. https://doi. org/https://doi.org/10.2309/java.12-2-11

20. Save your vein (s.f.). Recuperado en septiembre de 2020, de Save your vein: https://www.saveyourvein.org/consultado el 23/septiembre/2020

21. Koirala N, Anvari E, McLennan G. Monitoring and surveillance of hemodialysis access. Semin Intervent Radiol. 2016 Mar;33(1):25-30. doi: 10.1055/s-0036-1572548. PMID: 27013773; PMCID: PMC4806702.

22. Allon M, Robbin ML. Hemodialysis vascular access monitoring: current concepts. Hemodial Int. 2009 Apr;13(2):153-62. doi: 10.1111/j.15424758.2009.00359.x. PMID: 19432687; PMCID: PMC4017945.

23. Work $\mathrm{J}$. Role of access surveillance and preemptive intervention Semin Vasc Surg. 2011 Jun;24(2):137-42. doi: 10.1053/j.semvascsurg.2011.05.014. PMID: 21889103.

24. Ravani P, Palmer SC, Oliver MJ, Quinn RR, MacRae JM, Tai DJ, et al. Associations between hemodialysis access type and clinical outcomes: a systematic review. J Am Soc Nephrol. 2013 Feb;24(3):465-73. doi: 10.1681/ASN.2012070643. Epub 2013 Feb 21. PMID: 23431075; PMCID: PMC3582202.

25. Tal MG, Ni N. Selecting optimal hemodialysis catheters: material, design, advanced features, and preferences. Tech Vasc Interv Radiol. 2008 Sep;11(3):186-91. doi: 10.1053/j.tvir.2008.09.006. PMID:19100948.

26. Van Der Meersch $H$, De Bacquer D, Vandecasteele SJ, Van den Bergh B, Vermeiren P, De Letter J, et al. Hemodialysis catheter design and catheter performance: a randomized controlled trial. Am J Kidney Dis. 2014 Dec;64(6):902-8. doi: 10.1053/j.ajkd.2014.02.017. Epub 2014 Apr 6. Erratum in: Am J Kidney Dis. 2015 May;65(5):810. PMID: 24713222.

27. Li M, Zhang Z, Yu Y, Chen H, Li X, Ma J, et al. Clinical application of long-term palindrome catheter in hemodialysis patients. Iran J Kidney Dis. 2014 Mar;8(2):123-9. PMID: 24685735.

28. Siegel JB. Tunneled dialysis catheters: pearls and pitfalls. Tech Vasc Interv Radiol. 2008 Sep;11(3):181-5. doi: 10.1053/j.tvir.2008.09.005. PMID: 19100947

29. Guillermo-Corpus G, Ramos-Gordillo JM, Peña-Rodríguez JC. Survival and clinical outcomes of tunneled central jugular and femoral catheters in prevalent hemodialysis patients. Blood Purif. 2019;47(1-3):132-139. doi: 10.1159/000494206. Epub 2018 Oct 25. PMID: 30359982.

30. Brass P, Hellmich M, Kolodziej L, Schick G, Smith AF. Ultrasound guidance versus anatomical landmarks for internal jugular vein catheterization. Cochrane Database Syst Rev. 2015 Jan 9;1(1):CD006962. doi: 10.1002/14651858.CD006962.pub2. PMID: 25575244; PMCID: PMC6517109. 
Nefro Latinoam. 2021;18

31. Saugel B, Scheeren TWL, Teboul JL. Ultrasound-guided central venous catheter placement: a structured review and recommendations for clinica practice. Crit Care. 2017 Aug 28;21(1):225. doi: 10.1186/s13054-0171814-y. PMID: 28844205; PMCID: PMC5572160.

32. Jonczyk M, Althoff C, Slowinski T, Lieker I, Naik M, Auer J, et al. Urea-based recirculation validation of the symmetrical palindrome catheter. J Ren Care. 2017 Dec;43(4):242-246. doi: 10.1111/jorc.12210. Epub 2017 Aug 16. Erratum in: J Ren Care. 2018 Jun;44(2):123. PMID: 28815975.

33. Ling XC, Lu HP, Loh EW, Lin YK, Li YS, Lin $C H$, et al. A systematic review and meta-analysis of the comparison of performance among step-tip, split-tip, and symmetrical-tip hemodialysis catheters. J Vasc Surg. 2019 Apr;69(4):1282-1292. doi: 10.1016/j.jvs.2018.09.029. PMID: 30905366.

34. Castro V, Farber A, Zhang Y, Dicken Q, Méndez L, Levin SR, et al. Reasons for long-term tunneled dialysis catheter use and associated morbidity. J Vasc Surg. 2021 Feb:73(2):588-592. doi: 10.1016/j. jvs.2020.06.121. Epub 2020 Jul 21. PMID: 32707393. 